\title{
Re-operation of idiopathic full-thickness macular holes after initial surgery with internal limiting membrane peel
}

\author{
Mark J J D'Souza, ${ }^{1}$ Varun Chaudhary, ${ }^{2}$ Robert Devenyi, ${ }^{3}$ Peter J Kertes, ${ }^{4}$ \\ Wai-Ching Lam $^{5}$
}

${ }^{1}$ Department of Family Medicine, University of Western Ontario, London, Ontario, Canada

${ }^{2}$ Department of Surgery, St. Joseph Health Care, McMaster University, Hamilton, Ontario, Canada

${ }^{3}$ Toronto Western Hospital, University of Toronto, Toronto, Ontario, Canada

${ }^{4}$ The John and Liz Tory Eye Centre, Sunnybrook Health Sciences Centre, University of Toronto, Toronto, Ontario, Canada

${ }^{5}$ Department of Ophthalmology and Vision Sciences, University of Toronto, Toronto, Ontario, Canada

\section{Correspondence to}

Dr Wai-Ching Lam, Department of Ophthalmology, East Wing,

Room 6E 432, Toronto Western Hospital Toronto, Ontario,

Canada M5T 2S8;

waiching.lam@utoronto.ca

Accepted 30 January 2011 Published Online First 25 February 2011

\section{UNLOCKID}

This paper is freely available online under the BMJ Journals unlocked scheme, see http:// bjo.bmi.com/site/about/ unlocked.xhtml

\section{ABSTRACT}

Background/aims A retrospective consecutive case series to evaluate the efficacy of re-operation in patients with persistent or recurrent idiopathic full-thickness macular hole after initial surgery with internal limiting membrane peel (ILM).

Methods 491 patients underwent surgery for fullthickness macular hole from January 2004 to November 2007. Fifty-five patients either did not close or reopened during the follow-up period. Thirty patients with initial ILM peel underwent repeat surgery involving vitrectomy, enlargement of ILM rhexis and gas tamponade.

Results Anatomical closure rate was $88.8 \%$ for primary surgery and $46.7 \%(14 / 30)$ for re-operation. There was a statistically significant improvement in overall best corrected visual acuity (BCVA) from re-operation baseline BCVA $(p=0.02)$ within 1 year. For holes that did not close after the second surgery, visual acuity did not worsen.

Conclusion Re-operation has a reduced success rate of anatomical closure. However, BCVA is statistically significantly improved from re-operation baseline, so even though we cannot return vision to pre-pathological baseline, re-operation can improve on this new baseline.

\section{INTRODUCTION}

In 1991, Kelly and Wendel introduced a surgical procedure to close macular holes, a previously untreatable cause of poor and distorted central vision. By 1993, they had achieved an anatomical closure rate of $73 \%$ and a visual improvement rate for two or more lines of $55 \%{ }^{1}$ Retinal surgeons globally have been refining the technique for macular hole surgery ever since, and hole closure rates have been reported as exceeding $90 \%$ in some publications. ${ }^{2-8}$ Currently, the standard technique is pars plana vitrectomy with gas tamponade, with or without internal limiting membrane (ILM) peeling.

Many studies conclude that peeling the ILM improves hole closure rate, yet it remains somewhat controversial..$^{29-14}$ The primary objective of this study was to evaluate the efficacy of re-operation in patients with persistent or recurrent idiopathic full-thickness macular hole after initial surgery with ILM peel.

\section{MATERIALS AND METHODS Type of study}

A retrospective interventional consecutive case series was conducted.

\section{Patients}

The study population consisted of 491 consecutive full-thickness macular hole surgical cases from January 2004 to November 2007 from three retinal surgeons working at two tertiary care teaching hospitals in Toronto, Ontario, Canada. We reviewed all their records in this time period and did full chart reviews on patients who underwent re-operation.

\section{Data collection}

Data were collected by reviewing the charts that had macular hole surgery listed in the operating room booking records, and manually identifying the charts for patients that underwent a second macular hole operation. The patients' referring ophthalmologists or optometrists were called for more information if follow-up with their retinal surgeon was not of sufficient length. The following data were obtained: age; sex; estimated date of initial visual complaint, date of first and second operation; stage of the macular hole and posterior vitreous detachment (PVD) status before first operation; baseline best corrected visual acuity (BCVA) before first and second operations, and at 3 months, 6 months and 1 year after re-operation; baseline lens status before first operation and at BCVA after re-operation; macular hole configuration determined by optical coherence tomography (OCT) when available before re-operation as described by Hillenkamp et al; and fellow eye status with regards to macular hole. ${ }^{15}$

\section{Primary surgical technique}

The three retina surgeons involved performed macular hole surgery using a similar technique. It consisted of a standard three-port pars plana vitrectomy with induction of a PVD as appropriate. All procedures involved ILM peel with indocyanine green (ICG) dye. The three surgeons involved had different ICG staining techniques. Two of the surgeons mixed and diluted the ICG with a $5 \%$ dextrose solution, and injected it onto the posterior pole in a fluid-filled eye. The third surgeon mixed and diluted the ICG in Viscoat, Alcon, Inc, Huenenberg, Switzerland, and injected it onto the posterior pole with the infusion clamped. All three surgeons aspirated the ICG immediately after injection. All patients had gas tamponade with either non-expansile $\mathrm{C}_{3} \mathrm{~F}_{8}(14-15 \%)$ or $\mathrm{SF}_{6}(20 \%)$ tamponade. All patients were then instructed to lie face-down $90 \%$ of the time for 1 week. 


\section{Re-operation surgical technique}

The three retina surgeons performed a similar procedure. Re-operation began with a standard three-port pars plana vitrectomy, followed by enlargement of the ILM rhexis with adjunctive ICG staining. Autologous serum was used as an adjuvant in four of 30 cases. All patients then had gas tamponade with a non-expansile concentration of $\mathrm{C}_{3} \mathrm{~F}_{8}$ gas, and were subsequently instructed to lie face-down $90 \%$ of the time for 1 week.

\section{Statistical methods}

A paired two-tailed t test was performed with SAS software. Patients had to have data for at least two of the three time points ( 3 months, 6 months and 1 year post re-operation) to be included in the statistical analysis. Data were carried forward in the event of missing data. Counting fingers was translated as 0.01 and hand motions as 0.0033 in decimal Snellen notation.

\section{RESULTS}

There was no statistically significant difference between closed and unclosed populations in terms of sex, age at re-operation or time between surgeries. Further, we estimated the duration of the hole as the difference between re-operation date and the approximate date of initial visual complaint. Again, there was no statistically significant difference between closed and unclosed populations based on estimated duration of symptoms.

There was an $88.8 \%$ closure rate (436/491) for primary macular hole surgery in our study. All 25 patients that failed initial macular hole surgery and were not re-operated on declined re-operation either for personal reasons (a combination of difficulty with face-down positioning along with guarded prognosis) or deferred their decision to think about it and did not get back to their surgeon within a year of primary surgery. We, therefore, believe the conclusions of this paper apply to these patients as well. Five of 30 re-operated patients were excluded from the analysis because they did not have data for at least two of the three follow-up time points. Two patients underwent a third macular hole surgery within the 1-year period following re-operation for failure to close, and they were included in the analysis as intent to treat (figure 1).

There was a $46.7 \%$ success rate for re-operation $(n=30)$. Four of the 25 holes included in the statistical analysis (for meeting the requisite number of follow-up time points) reopened after initial macular hole surgery; only one out of the four (25\%) closed following re-operation. We strictly defined reopening as macular hole closed on examination at 3 months, and found to

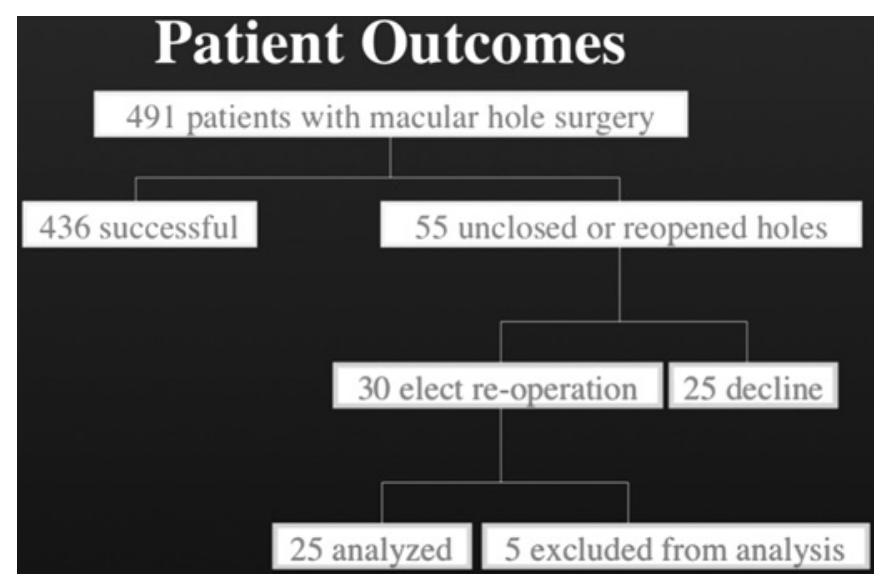

Figure 1 Patient outcomes. be open thereafter. Twenty-one of the 25 macular holes included in the analysis were unclosed after initial operation; eleven of these 21 closed (52\%) after re-operation.

There was a statistically significant improvement between overall BCVA and re-operation baseline $(p=0.020)$ (figure 2). Overall BCVA takes each patient's highest visual acuity at any point within 1-year postoperatively. Examining only patients who achieved anatomical closure following re-operation, this improvement was also significant $(n=12, p=0.038)$ (figure 3 ). Examining only patients whose holes did not close after reoperation ( $n=13)$, their visual acuity did not decrease (figure 4).

Of our 13 patients with optical coherence tomography (OCT) prior to re-operation, seven had cuffs of subretinal fluid as per Hillenkamp et al, and six did not. ${ }^{15}$ Anatomical closure was achieved in $71 \%$ of those with this cuff and only $50 \%$ of those without.

At overall BCVA measurement for our 25 analysed patients, 13 were classified as pseudophakic, four had cataract formation and eight did not have lens status recorded on chart review. Of the four patients with cataracts, one had 1+nuclear sclerosis (NS), one had $2+\mathrm{NS}$ and two had $3+\mathrm{NS}$.

\section{DISCUSSION}

Despite the high success rate of macular hole surgery, it is not $100 \%$. There have been many studies dedicated to this subset of macular hole patients who fail to close. The current success rate of primary surgery is $80-95 \% .^{2-8}$ Valldeperas et al reported a retrospective case series of 532 patients who underwent macular hole surgery, but with only the rare ILM peel. Re-operation on 52 unclosed holes yielded a $76 \%$ anatomical closure rate. Re-operation on 21 reopened holes yielded a $100 \%$ anatomical closure rate. They defined reopened as holes that appeared closed on examination, but then were found to be open again at a later date. This reopening typically took place several months later. ${ }^{16}$

Ours is the largest case series on macular hole re-operation on a patient population whose ILM was routinely peeled during primary surgery. The $88.8 \%$ success rate of primary surgery with this technique is consistent with that reported elsewhere in the literature on primary macular hole surgery. ${ }^{2-8}$ Re-operation with this technique yields a reduced anatomical success rate of hole closure $(46.7 \%, \mathrm{n}=30)$ compared with primary surgery. Note that only 25 of the 30 re-operated patients were included in the analysis because the other five did not have data for at least two out of three of the follow-up time points. We achieved a $52 \%$ closure rate with re-operation of unclosed holes $(n=21)$ and $25 \%$ for reopened holes $(n=4)$. These are both much lower

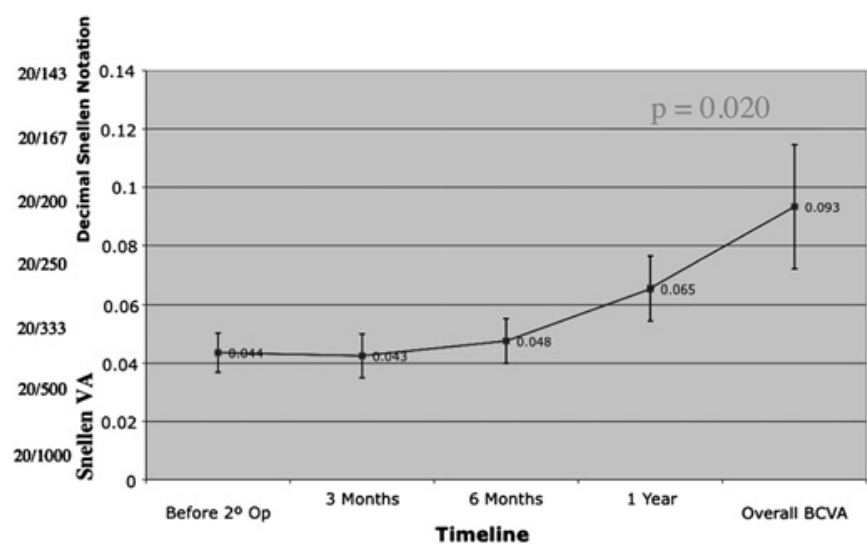

Figure 2 Best corrected visual acuity (BCVA) for all patients. VA, visual acuity. 


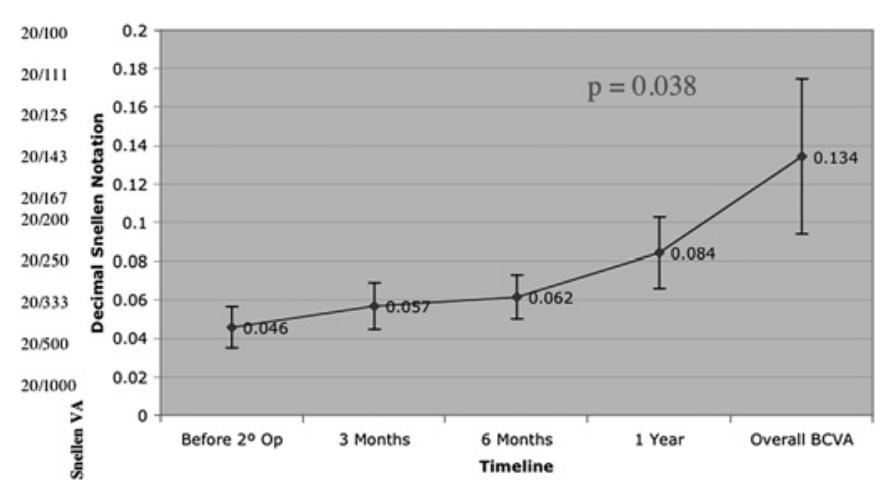

Figure 3 Best corrected visual acuity (BCVA) for patients with anatomical closure.

than the figures achieved by Valldeperas et al, which we attribute to difference in technique. In their study, ILM peeling was rarely carried out in both initial surgery and re-operation. We, therefore, hypothesise that re-operation is much less likely to succeed in patients whose ILMs have already been peeled; there is little else to do when the posterior hyaloid has been detached and the ILM removed.

Of importance, overall BCVA was significantly improved from re-operation baseline. It is also noteworthy that repeat surgery did not worsen overall BCVA in the subset of patients whose macular holes remained. Overall BCVA takes each patient's highest visual acuity at any point within 1 year postoperatively. This approach was taken to guard against confounding factors such as cataract progression, as macular hole surgery accelerates cataract formation. ${ }^{16}$ For example, a patient with successful re-operation may have a decrease in visual acuity from 6 months to 1 year postoperatively solely due to a new surgery-induced cataract. Clinically, however, $88 \%$ of patients (22 of 25) did not improve by at least one Snellen line; $8 \%$ (two of 25 ) improved by two lines and $4 \%$ (one of 25 ) improved by four lines. We hypothesise that the poor visual acuity outcomes relative to primary surgery relate to the added duration of the hole, and having to wait for and undergo a second surgery. It is often difficult to ascertain how long a hole has been present. Second, we know that longer duration holes tend to be bigger and less likely to close, so the ones that have failed may already be predisposed to worse outcomes. Moreover after the ILM has been peeled, residual ILM is more adherent. Additional surgical trauma damages retinal pigment epithelium and photoreceptors,

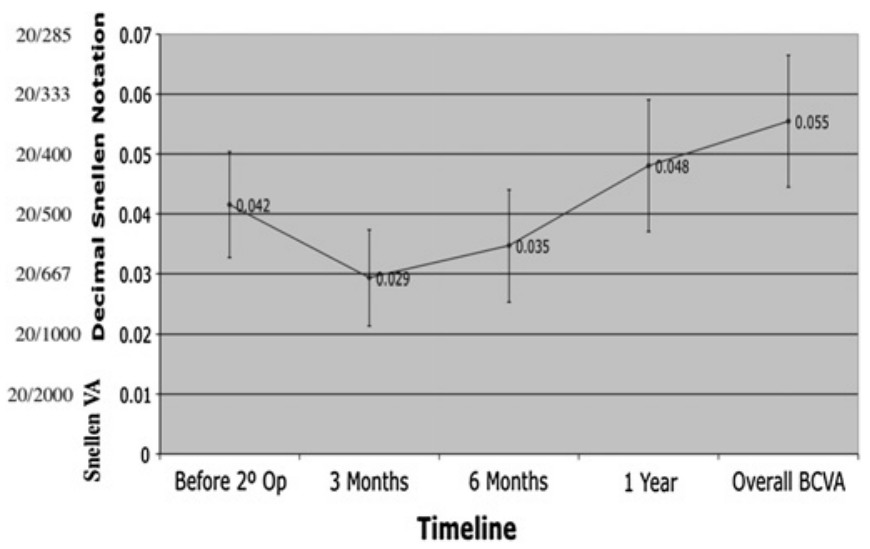

Figure 4 Best corrected visual acuity (BCVA) for patients that did not achieve anatomical closure. VA, visual acuity. and is probably an important component of disappointing visual results.

While estimated duration of macular hole was recorded, it is unreliable so it was not analysed as a variable with respect to macular hole prognosis. Patients are often inaccurate in dating the onset of symptoms. Macular holes are usually a monocular disease process, so patients may not notice symptoms for some time.

Hillenkamp et al performed a retrospective consecutive case series of 28 unclosed macular holes. Each patient underwent OCT prior to the repeat macular hole surgery. An elevated cuff of subretinal fluid at the margin of the macular hole (and overlapping it) was found to be a strong prognostic indicator for both anatomical closure $(p=0.001)$ and BCVA improvement $(p=0.048)$. This is contrasted with a flat, punched out hole with no distinct retinal cuff on OCT. To account for these findings, it was proposed that there is lack of adhesion between the macular hole margin and the underlying retinal pigment epithelium in cases with an elevated cuff of subretinal fluid at the margin of the macular hole. This may facilitate closure of the macular hole. This assumes that closure involves the centripetal movement of retinal tissue to occupy the foveal region ${ }^{15}$ (figure 5). OCT was not done routinely preoperatively in our patient population. So while our limited data supports Hillenkamp's conclusion, that this cuff of subretinal fluid is a prognostic factor in macular hole surgery, our small sample size of 13 patients with OCTs does not allow us to draw any definitive conclusions.

ILM peeling began in 1992 in the belief that it would help prevent the reopening of macular holes. ${ }^{10}$ It is believed that the ILM acts as a scaffold for contractile tissue to exert tangential traction on macular holes, leading to reopening. ${ }^{2}$ In light of the hypothesis that macular holes are closed by glial cell proliferation, Sheidow et al also propose that a surgeon can induce a relatively consistent degree of trauma to facilitate this gliosis over a macular hole by utilising ILM peeling. ${ }^{7}$ An additional benefit is that the removal of ILM also reduces the chance of epiretinal membrane formation. ${ }^{14}$

Moreover, we hypothesise that with ILM peeling, all vitreal traction should be removed. The majority of failures with our technique were unclosed holes $(76.9 \%)$. The causal mechanism of these holes that reopened remains unclear. We believe that elevation of the posterior hyaloid and removal of the ILM, as can

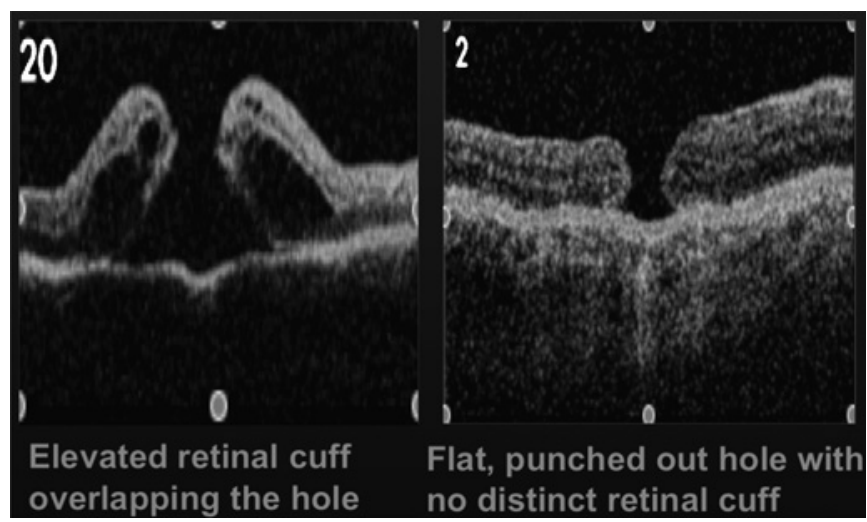

Figure 5 The optical coherence tomography (OCT) image on the left shows an elevated cuff of subretinal fluid at the margin of the macular hole. According to Hillenkamp et al, it was found to be a strong prognostic indicator for both anatomical closure $(p=0.001)$, and best corrected visual acuity improvement $(p=0.048)$. This is in comparison to the OCT image on the right of a flat, punched out macular hole with no distinct retinal cuff. Reproduced with permission from Hillenkamp $\mathrm{J}$, et al. ${ }^{15}$ 
reliably and reproducibly be done with intravitreal triamcinolone and ICG staining respectively, not only removes the centripetal traction that is present, but also ensures that any residual vitreous adherent to the retinal surface is removed. Certainly the difficulties of face-down positioning and non-compliance are likely to be important contributors, as may be hole size and presence of the hole for longer than is realised.

The weaknesses of this study include the retrospective nature of the data collection, involving three surgeons, and not having data for hole size as preoperative OCTs were not universally performed. Further, cataracts present a significant cofounder for BCVA. ${ }^{17}$ Moreover, as some of the patients declined a re-operation, this study had a limited sample size with limited statistical power.

Ultimately, the decision to proceed with repeat macular hole surgery must be done after careful deliberation with the patient, informing the patient of the benefits, risks, and the likelihood of hole closure and visual acuity improvement. We hope that our study will help inform this decision-making process when considering re-operation after failed macular hole surgery with ILM peel.

Funding None.

Competing interests None declared.

Ethics approval Sunnybrook Health Sciences Centre (Research Ethics Office project number 256-2009) and University Health Network (Research Ethics Board project number 09-0508-AE).

Provenance and peer review Not commissioned; externally peer reviewed.

\section{REFERENCES}

1. Wendel RT, Patel AC, Kelly NE, et al. Vitreous surgery for macular holes. Ophthalmology 1993;100:1671-6.
2. Brooks J, Logan $\mathrm{H}$. Macular hole surgery with and without internal limiting membrane peeling. Ophthalmology 2000;107:1939-49.

3. Christmas NJ, Smiddy WE, Flynn HW Jr. Reopening of macular holes after initially successful repair. Ophthalmology 1998;105:1835-8.

4. Gross JG. Late reopening and spontaneous closure of previously repaired macular holes. Am J Ophthalmol 2005;140:556-8.

5. Kusaka S, Sakagami K, Kutsuna M, et al. Treatment of full-thickness macular holes with autologous serum. Jpn J Ophthalmol 1997:41:332-8.

6. Paques M, Chastang C, Mathis A, et al. Effect of autologous platelet concentrate in surgery for idiopathic macular hole: Results of a multicenter, double-masked, randomized trial. Platelets in Macular Hole Surgery Group. Ophthalmology 1999:106:932-8.

7. Sheidow TG, Blinder KJ, Holekamp N, et al. Outcome results in macular hole surgery: An evaluation of internal limiting membrane peeling with and without indocyanine green. Ophthalmology 2003;110:1697-701

8. Vine AK, Johnson MW. Thrombin in the management of full thickness macular holes. Retina 1996;16:474-8.

9. Da Mata AP, Riemann CD, Nehemy MB, et al. Indocyanine green-assisted internal limiting membrane peeling for macular holes to stain or not to stain? [see comment] Retina 2005;25:395-404.

10. Rezende FA, Kapusta MA. Internal limiting membrane: ultrastructural relationships, with clinical implications for macular hole healing. Can J Ophthalmol 2004:39:251-9.

11. Stec LA, Ross RD, Williams GA, et al. Vitrectomy for chronic macular holes. Retina 2004;24:341-7.

12. Terasaki H, Miyake $Y$, Nomura R, et al. Focal macular ERGs in eyes after removal of macular ILM during macular hole surgery. Invest Ophthalmol Vis Sci 2001;42:229-34.

13. Uemoto R, Yamamoto $\mathrm{S}$, Aoki T, et al. Macular configuration determined by optical coherence tomography after idiopathic macular hole surgery with or without internal limiting membrane peeling. Br J Ophthalmol 2002;86:1240-2.

14. Yoshida M, Otsubo A, Kishi S. Incidence of reopening of macular hole after successful surgery with removal of internal limiting membrane. Japanese Journal of Clinical Ophthalmology 2002;56:999-1003.

15. Hillenkamp J, Kraus J, Framme C, et al. Retreatment of full-thickness macular hole: Predictive value of optical coherence tomography. Br J Ophthalmol 2007;91:1445-9.

16. Valldeperas $\mathbf{X}$, Wong D. Is it worth reoperating on macular holes? Ophthalmology 2008;115:158-63.

17. Vote BJ, Membrey WL, Casswell AG. Autologous platelets for macular hole surgery: the Sussex Eye Hospital experience. Clin Experiment Ophthalmol 2004;32:472-7. 